\title{
Time-Lapse Observation of the Deformation of Polyethylene Lamellae by AFM
}

\author{
Rebecca C. Savage ${ }^{1,2}$, Jamie K. Hobbs ${ }^{1}$ \\ ${ }^{1}$ University of Sheffield, Sheffield, United Kingdom \\ ${ }^{2}$ Currently working for ExxonMobil Chemical Company, Houston, USA
}

Semicrystalline polymers consist of sheet-like crystalline lamellae with a thickness of $\sim 10 \mathrm{~nm}$ interspaced with disordered amorphous material. These two phases are bound together by the polymer chains which run through both, and it's this complicated structure that give plastics many of their desirable properties, such as toughness and strength. However, it also complicates the study of their behaviour during deformation. Most of our current understanding is based on scattering and spectroscopy but these measure the average behaviour of many molecules and lamellae. Increasingly more microscopy studies are being performed but typically these do not follow the evolution of single structures [1, 2] or do so at a scale greater than a few microns [3, 4]. Atomic Force Microscopy (AFM) is ideally suited for studying materials at the length-scale of polymer lamellae and we have applied it to follow individual lamellae in a polyethylene film as they undergo tensile strain and observe the various deformation mechanisms taking place.

The sample used was a commercial high density polyethylene film, $20 \mu \mathrm{m}$ thick. It was determined by GPC to be of medium molecular weight with a relatively broad distribution $\left(M_{w} 141,000, M_{w} / M_{n}=10\right)$. The sample was cut into a dog-bone shape and sonicated in acetone prior to imaging. AFM images were taken at room temperature on a Dimension 3100 (Bruker, Santa Barbara, USA) AFM in tapping mode with a Bruker TESPA-V2 cantilever $(\mathrm{k} \sim 42 \mathrm{~N} / \mathrm{m})$. A tensile strain rack was made in-house to fit under the AFM head. After strain was applied the sample was allowed to relax before imaging. The strain could be monitored in the area immediately around the area of interest via the AFM optics. As it is the small-scale processes that are the primary focus of this work the strain values reported here are those observed by the AFM optics, rather than between the bulkheads, and is defined as the engineering strain $\varepsilon=\left(l-l_{0}\right) / l_{0}$.

AFM images of the unstrained sample show a highly crystalline structure, broadly oriented perpendicularly to the strain direction but far from the long-range order found in oriented polyethylene. The lamellae were $\sim 20 \mathrm{~nm}$ wide, oriented with both short edge up and flat side up on the surface, and were typically arranged in stacks of 3 4 separated by disordered material (Figure 1). When strain was applied to the sample the lamellar stacks move apart (Figure 2a,b). At around the film's yield point crazes begin to open (Figure 2b), spanned by fibrils, and these gradually widen as the strain is increased. At the same time the stacks frequently bend to form chevrons (Figure 2c,d). At high strains some of the lamellae at the edge of the stacks break (Figure 2d, arrowed). Furthermore, some of the gaps between the fibrils widen and appear to be crossed by smaller fibrils $\sim 12 \mathrm{~nm}$ wide (Figure $2 \mathrm{c}$, arrowed).

In other areas of the image lamellae oriented with the flat side up can be seen to slip over each other at small strains (Figure 2). At higher strains some of these surfaces begin to take on a rough appearance (Figure 2e-h), possibly fragmentation of the lamellae. Another indication of lamellar fragmentation are edge-on lamellae oriented with their plane in the strain direction, in which discontinuities begin to appear at $\varepsilon \sim 40 \%$ and subsequently break into increasingly smaller pieces as the strain increases. 
In conclusion we wish to show that the AFM can be a useful tool for characterizing materials with complicated nanoscale structures, such as semicrystalline polymers, as they undergo deformation. Observing the individual deformation mechanisms will allow us to gain a greater understanding of how nanoscale morphology relates to macroscale properties [5].

\section{References:}

[1] A. Rozanski, A. Galeski, Macromolecules, 44 (2011), p. 7273.

[2] C. Thomas et al, Polymer, 48 (2007), p. 6041.

[3] A. Opdahl, G.A. Somorjai, J. Poly. Sci. B, 39 (2001), p. 2263.

[4] C. Thomas et al, Polymer, 50 (2009), p. 3714.

[5] The authors acknowledge funding from the EPSRC, and would like to thank Nic Mullin, Chris Holland and Michael Wier (University of Sheffield) for their help and contributions to this work.
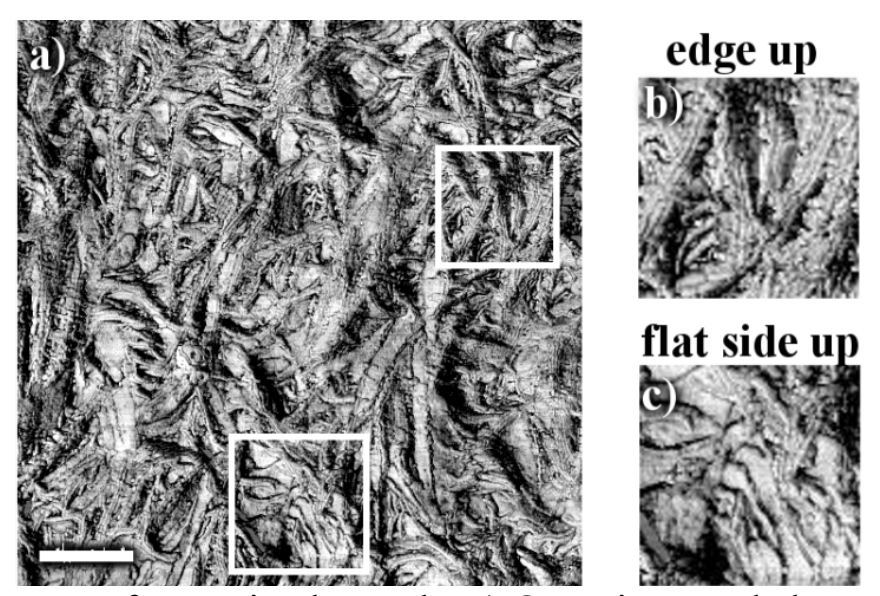

Figure 1. AFM phase images of unstrained sample. a) Overview, scale bar $500 \mathrm{~nm}$. b,c) Details from marked areas of (a) showing b) edge-on lamellae and c) flat-on lamellae. b,c) Images $500 \mathrm{~nm}$ wide

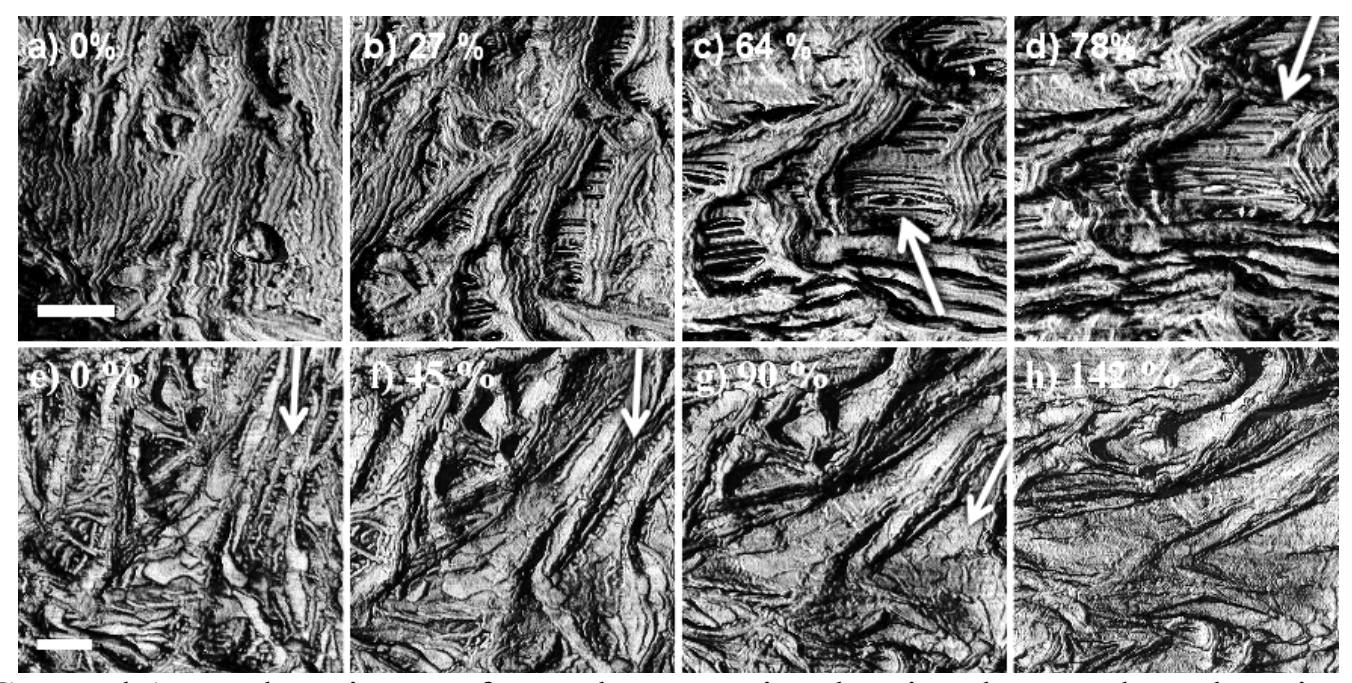

Figure 2. Cropped AFM phase images from a longer series showing the sample undergoing strain. a-d) Crazes opening between edge-on lamellae. e-h) Flat-on lamellae initially slipping over (e,f) each other before beginning to fragment $(\mathrm{g}, \mathrm{h})$. Scale bars are $200 \mathrm{~nm}$, the strain is applied horizontally. 